Pulsar Astronomy …2000 and Beyond

ASP Conference Series, Vol. 202, 2000

M. Kramer, N. Wex, and R. Wiclcbinski, eds.

\title{
A Fast Search Technique for Binary Pulsars
}

\author{
Scoti M. Ransom \\ Harvard-Smithsonian Center for Astrophysics, 60 Garden St., MS 10, \\ Cambridge, MA 0D138, USA
}

\begin{abstract}
I describe a computationally simple, efficient, and sensitive method to search long observations for pulsars in binary systems. The technique looks for orbitally induced sidebands in the power spectrum around a nominal spin frequency, enabling it to detect pulsars in high-or low-mass binaries with short orbital periods $\left(P_{\text {orb }} \lesssim 5 \mathrm{~h}\right)$.
\end{abstract}

Pulsars in binary systems experience orbital accelerations which Doppler shift the pulsar spin frequencies. This Doppler smearing, and the huge parameter space we must scarch because of it, makes the discovery of all but the longestperiod binaries virtually impossible (e.g., Johnston \& Kulkarni 1991).

The new technique, which I call phase modulation searching, greatly reduces the size of the search parameter space, with only a modest decrease in sensitivity compared to a fully coherent search. These searches rely on the fact that if the observation time is longer than the orbital period $\left(T_{o b s}>P_{o r b}\right)$ the orbital Doppler shifts effectively phase modulate the pulsar spin frequency.

Phase modulation results in a family of sidebands, evenly spaced by the orbital period, around the intrinsic pulsar signal in the frequency domain. These sidebands are effectively short periodic sequences in the full power spectrum and are detectable using short FFTs or sums of powers. When $T_{o b s}>P_{o r b}$ these periodic sidebands occur for all combinations of Keplerian orbital elements, which reduces the binary portion of the search parameter space to only 2 parameters: the modulation period, $P_{o r b}$, and amplitude, $\Phi_{\text {orb }}=2 \pi x f_{p s r}$ ( $x$ is the projected semi-major axis of the orbit and $f_{p s r}$ is the pulsar spin frequency).

The simplicity and efficiency of phase modulation searching allows the analysis of very long time-series (e.g., satellite observations of a source over many days or year-long data sets from gravitational wave detectors) which greatly improves the chances of detecting weaker sources. Data analysis using these and other advanced Fourier techniques is ongoing on several long radio observations of globular clusters (see Figure 1) and on various RXTE observations.

Acknowledgments. I would like to thank both Steve Eikenberry and Jim Cordes for useful ideas and discussions related to this work.

\section{References}

Johnston, H. M. \& Kulkarni, S. R. 1991, ApJ, 368, 504

Nice, D. J. \& Thorsett, S. E. 1992, ApJ, 397, 249

Ransom, S. M., Cordes, .J. M. \& Eikenberry, S. S. 2000, in preparation. 

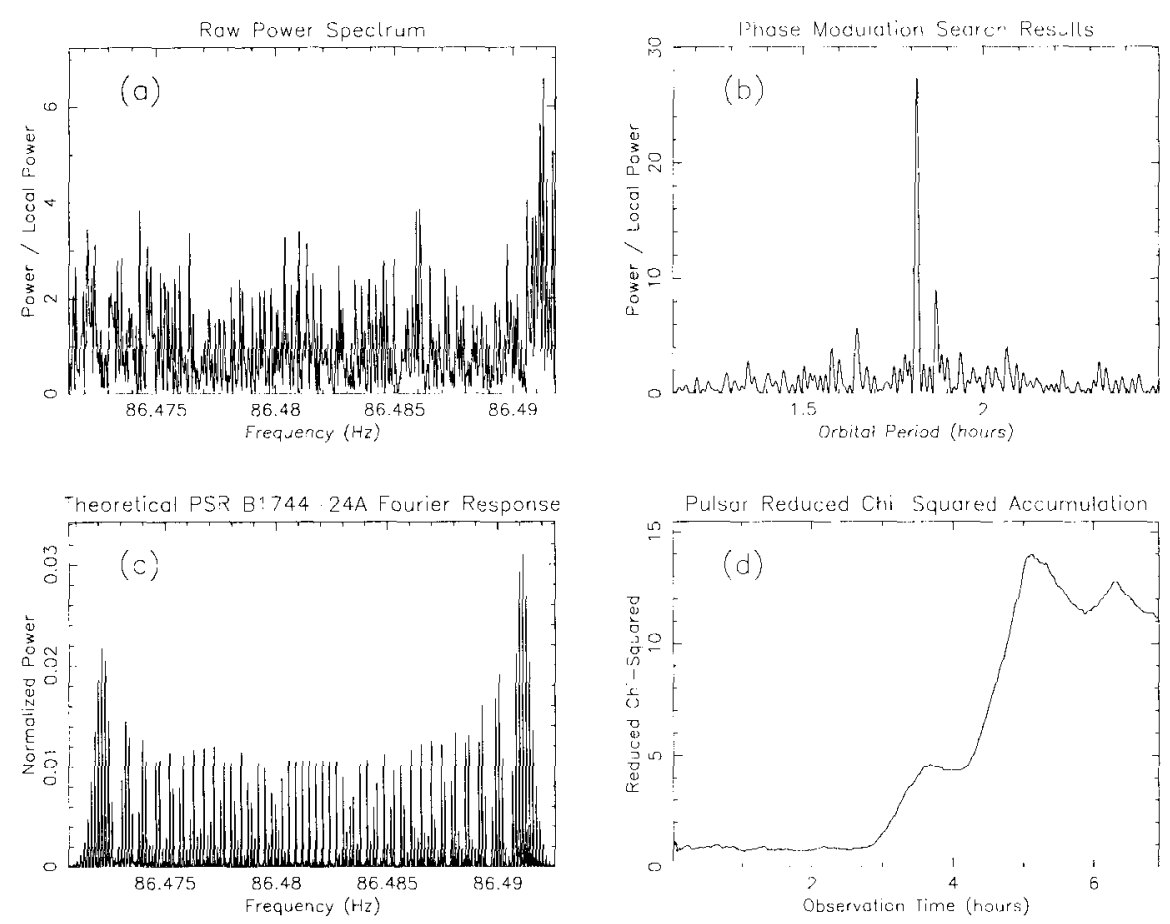

Figure 1. Results from a phase modulation search of a 7 hour observation of the globular cluster Terzan5 taken with the Parkes Multibeam system. (a) shows a section of the power spectrum from the full observation centered on the spin frequency of the eclipsing and "disappearing" binary millisecond pulsar PSR B1744-24A $\left(P_{\text {orb }}=1.82 \mathrm{~h}\right)$ (see Nice \& Thorsett 1992). All power levels are consistent with being due to noise. (b) shows the $\sim 7 \sigma$ detection of the pulsar by taking a short FFT of (a). Note that we detect the pulsar at the accurately determined orbital period instead of the spin period. The detection results from the fact that buried beneath the noise in (a) are the periodic sidebands caused by the orbital phase modulation of the pulsar spin frequency. (c) shows what these sidebands would look like for PSR B1744-24A with no noise if it were present during the full observation. The total number of sidelobes is approximately equal to $2 \Phi_{\text {orb }} \sim 130$. (d) shows the accumulation of reduced $\chi^{2}$ while folding the pulsar signal at the known ephemeris. The pulsar is "on" for only $\sim 2$ non-continuous hours during the observation. The fact that we detect the pulsar using a phase modulation search, even though the orbit only "modulates" the spin frequency for approximately one noncontinuous orbital period, demonstrates the robustness of the search technique. If the pulsar had been present during the full observation, the sidelobes would have been sharper and stronger, resulting in a much more significant detection. 